\title{
Shape Description of 3D Objects by Curvature Spin Images Generated via Gaze Modeling
}

\author{
Takashi Nakamae, Makoto Maeda and Katsuhiro Inoue \\ Kyushu Institute of Technology, 680-4 Kawatsu, Iizuka, Fukuoka 820-8502, Japan \\ E-mail: \{nakamae@kiri.ces, maeda@ces, inoue@ces\}.kyutech.ac.jp
}

\begin{abstract}
To realize a model-based 3D object recognition, we propose a feature extraction method and a shape descriptor using the geometric features. First, the feature extraction method based on a novel gaze modeling is proposed. In the modeling process, the surface model is independently estimated for a part of range data restricted by several gaze domains. Hence, since the features are independently extracted from each gaze domain, inconsistent or incorrect features may be obtained. Therefore a stochastic method that enables us to integrate such features by evaluating the reliability of each gaze model is introduced. Next a shape descriptor, curvature spin image, is proposed. The CSI is created based on the ratio of surface curvatures. The main contribution of this paper is experimental analysis of the use of CSIs with various tuning parameters.
\end{abstract}

\section{Introduction}

Recently range data has been widely used for many applications, since it provides 3D geometrical information about objects. Especially, the entire circumferential range data that can be produced by synthesizing several range images obtained from different viewpoints is worth utilizing, since it enables us to acquire 3D geometrical complete information about the circumferential shape of an object. To realize 3D object recognition using such range data is one of central issues in computer vision. Since it is, however, a set of simple 3D points, geometric relations among these points are ambiguous. In order to apply it for 3D shape analysis and object recognition, hence, 3D modeling techniques are required to represent the $3 \mathrm{D}$ shape as surface models.

The 3D object recognition that is achieved by matching an observed model in a scene with stored object models is called model-based object recognition $[1,2]$. In the technique, some geometric features such as feature points, surface curvatures and surface normal are extracted from each model, so that both the object model and the observed scene can be represented by sets of these features. Hence, the recognition of an object in a scene amounts to the discovery of a match between a subset of the scene features and a subset of the features of some object models stored in memory.
In general, it is not so easy to perform the shape modeling for range data of various objects in complex scene. Shape representations that are described by such features are required so that surface models can be compared efficiently. The central issues of the recognition system are how the features can be efficiently extracted from the shape models and how 3D shape should be described using the features.

In this paper, a feature extraction method based on a novel gaze modeling is proposed. In the modeling process, the surface model is independently estimated for a part of range data restricted by several gaze domains. Hence, since the features are independently extracted from each gaze domain, inconsistent or incorrect features may be obtained. Therefore a stochastic method that enables us to integrate such features by evaluating the reliability of each gaze model is introduced. So that, even though the range data of complex objects is given, the method enables us to obtain the geometric features as accurately as possible.

On the other hand, there is Spin Image as one of well-known 3D shape descriptors[1]. The spin image is a data level shape descriptor that is used to match surfaces represented as surface meshes. Hence, a model is represented as a set of descriptive images that efficiently encode global properties of the surface using an object-centered coordinate system.

In this paper, a shape descriptor that is similar to spin image is proposed. It is named curvature spin image (CSI) after the spin image. In Spin Image, surface shape is described by a dense collection of $3 \mathrm{D}$ points and surface normals. However, since spin images are created based on surface normals estimated at vertices, the estimation accuracy may affect the matching between spin images. In contrast, the CSI is created based on the ratio of surface curvatures. The main contribution of this paper is experimental analysis of the use of CSIs with various tuning parameters.

\section{Gaze Modeling}

\subsection{Gaze Domains}

Given a set of range data of an object, we consider $K$ gaze domains in order to restrict gaze fields to local parts of the object. Fig.1 shows the diagram of a gaze domain. Given a center point $\boldsymbol{P}$ and the direction $-\boldsymbol{n}$ 


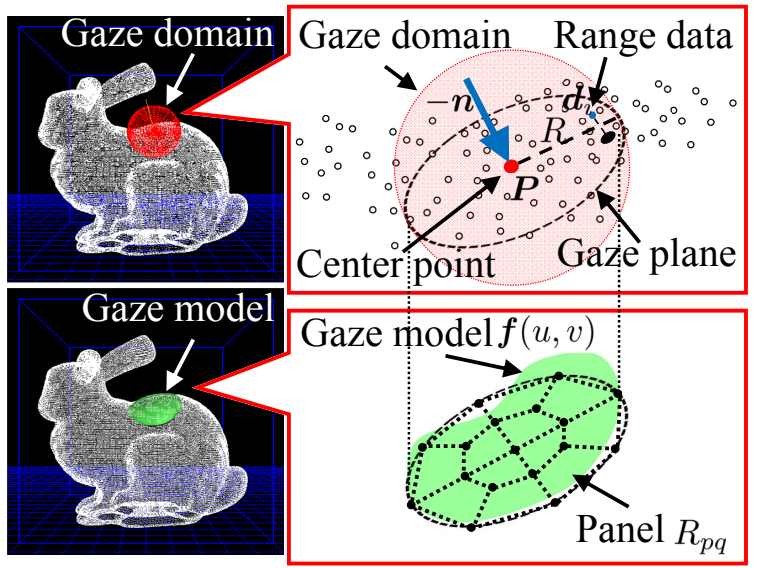

Fig. 1: The diagram of a gaze domain which includes range data that is a part of a rabit and the gaze model estimated in the gaze domain.

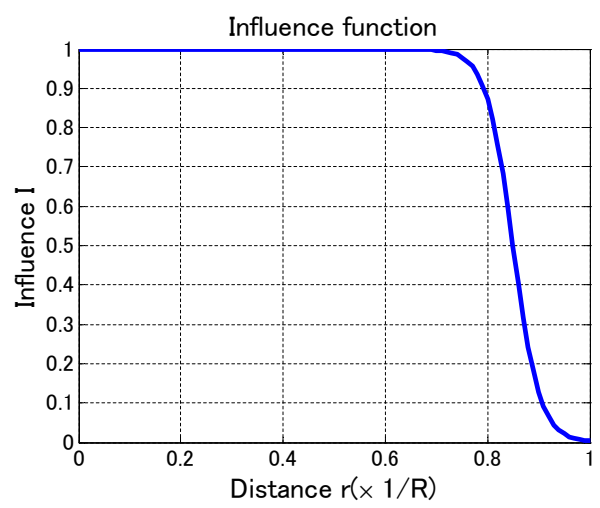

Fig. 2: The diagram of function that provides influence indices $I_{i}$.

of gaze, the gaze domain is defined as a sphere with the radius $R$. That is, gazing assumes that the point $\boldsymbol{P}$ and its vicinity within $R$ on an object is paid attention to.

Given the range data of an object, we define $D_{k}$ as a subset of the range data that is inside the $k$ th gaze domain. Each data point $\boldsymbol{d}_{i}:\left(x_{i}, y_{i}, z_{i}\right) \subseteq D_{k}$ is defined with the normal vector $\boldsymbol{n}_{i}$ that is estimated by using its 2 neighbor points. Furthermore, an influence index $I_{i}$ is given depending on the distance $r_{i}=\left\|\boldsymbol{d}_{i}-\boldsymbol{P}\right\|$ by using a function as shown in Fig.2. It is used to determine reliabilities of panels that are mentioned later.

\subsection{Gaze Modeling}

For the range data restricted by the $k$ th gaze domain $(k=1,2, \cdots, K)$, a parametric surface model $\boldsymbol{f}_{k}(u, v)$ that is defined as 4 th order NURBS surface can be estimated by using a regularization approach in order to extract 3D features of the object. More complete description of the regularization approach is given in [4]. We have chosen the name gaze modeling.

Let the panels $R_{p q}$ be regions which are divided by the knots in $u-v$ parameter space, that is equivalent to divide the gaze plane into $p \times q$ regions. Then the mean square error $\sigma_{R_{p q}}^{2}$ inside each panel $R_{p q}$ is computed in order to determine the reliability $S_{p q}$ of the panel $R_{p q}$. When a point $\boldsymbol{d}_{i}$ is corresponding to the panel $R_{p q}, S_{p q}$ is updated using its influence index $I_{i}$ as follows.

$$
S_{p q}= \begin{cases}S_{p q}+I_{i} & \left(\boldsymbol{n}_{i} \cdot \boldsymbol{n} \geq 0\right) \\ S_{p q}-I_{i} & \left(\boldsymbol{n}_{i} \cdot \boldsymbol{n}<0\right)\end{cases}
$$

The reliabilities $S_{p q}$ have been determined when the above updating procedure is repeated for all data points $\boldsymbol{d}_{i} \subseteq D_{k}$, where $S_{p q}$ is set to 0 if $S_{p q}<0$.

As mentioned above, for each gaze domain, the surface model $\boldsymbol{f}_{k}(u, v)$ and the reliabilities $S_{p q}$ of the panels $R_{p q}$ are obtained.

\subsection{Features Extraction}

Since the surface models $\boldsymbol{f}_{k}(u, v)$ have been obtained from the $K$ gaze domains, this section provides how to extract features from the surface models.

\subsubsection{Curvature features}

In general, a surface is characterized by its intrinsic differential properties. Differential geometry provides several measures of curvature, which include Gaussian and mean curvatures, or principal curvatures as their equivalents. Once the partial derivatives of $\boldsymbol{f}_{k}(u, v)$ are obtained, the curvatures can be easily computed[3]. The curvatures are direction-free quantities. These are invariant to arbitrary transformation of the $u-v$ parameters as well as rotations and translations of a surface. Combination of these curvature measures enables the local surface type to be categorized.

\subsubsection{Features integration based on mixture model}

Since the features are independently extracted from the each gaze domain, inconsistent or incorrect features may be obtained. Some residuals may also exist depending on the measurement noise and the complexity of 3D shape, although the surface model is fitted to the $3 \mathrm{D}$ data points as accurately as possible. Therefore, we propose a novel method for integrating such various features extracted from the gaze domains. It is realized by a stochastic approach based on a mixture model that treats the gaze domains as clusters, so that it enables us to evaluate the reliability of the obtained features. Finally, we can obtain robust and consistent features via whole gaze domains.

Now, let a random variable $X$ be the difference between a data point $\boldsymbol{d}$ and a surface model $\boldsymbol{f}(u, v)$. Then, the distribution of $X$ can be represented as the following mixture model,

$$
\begin{aligned}
p(X) & =\sum_{k=1}^{K} \operatorname{Pr}\left(C_{k}\right) p\left(X / C_{k}\right) \\
& =\sum_{k=1}^{K} \operatorname{Pr}\left(C_{k}\right) \sum_{p} \sum_{q} \operatorname{Pr}\left(R_{p q}\right) p\left(X / R_{p q}, C_{k}\right),
\end{aligned}
$$


where $\operatorname{Pr}\left(C_{k}\right)$ denotes a priori probability of the gaze cluster $C_{k} . \operatorname{Pr}\left(C_{k}\right)$ is defined as the rate of the number of data points included in this gaze cluster $C_{k}$ for the total number. $\operatorname{Pr}\left(R_{p q}\right)$ is defined as $S_{p q} / \sum_{m} \sum_{n} S_{m n}$.

If the data points $\boldsymbol{d}_{i} \subseteq D_{k}$ are fitted as accurately as possible to the surface model $\boldsymbol{f}_{k}(u, v)$ that is defined in the gaze cluster $C_{k}$, then $\mathrm{X}$ for the points can be almost 0 and become various depending on the noise and the complexity of shape. That enables us to establish the following assumption, since the mean square error $\sigma_{R_{p q}}^{2}$ inside each panel $R_{p q}$ has been computed. On the condition that a subset of data points $\boldsymbol{d}_{i} \subseteq D_{k}$ belongs to the panel $R_{p q}$, the distribution $p\left(X / R_{p q}, C_{k}\right)$ that the difference $\mathrm{X}$ between the data points and the cluster $C_{k}$ represents is assumed to be normal distribution $\mathcal{N}\left(0, \sigma_{R_{p q}}^{2}\right)$.

From the above formulation, we can obtain a posteriori probability $\operatorname{Pr}\left(C_{k} / X_{i}\right)$.

$$
\operatorname{Pr}\left(C_{k} / X_{i}\right)=\frac{p\left(X_{i} / C_{k}\right) \operatorname{Pr}\left(C_{k}\right)}{\sum_{j=1}^{K} p\left(X_{i} / C_{j}\right) \operatorname{Pr}\left(C_{j}\right)}
$$

Given a point $\boldsymbol{d}_{i}$ sampled on the surface that is defined in a gaze cluster $C_{k}$, The $3 \mathrm{D}$ shape features of the point are computed with the probability $\operatorname{Pr}\left(C_{k} / X_{i}\right)$. Simultaneously, a point near the sampled point may be sampled on the surface that is defined in the other gaze cluster $C_{l}$. The features of the point are also computed with the probability $\operatorname{Pr}\left(C_{l} / X_{i}\right)$. However, the features extracted from the different clusters may be inconsistent or incorrect. In order to integrate such features, we try to evaluate reliabilities of the features by comparing the probability $\operatorname{Pr}\left(C_{k} / X_{i}\right)$ with $\operatorname{Pr}\left(C_{l} / X_{i}\right)$. Consequently, we can select the robust and reliable features by the reliabilities even though there are the inconsistent or incorrect.

\section{Shape Description of 3D Objects}

This section provides how to describe 3D shapes using the reliable curvature features. We propose a shape descriptor, curvature spin image (CSI). In this approach, we attempt to represent a surface model as a set of descriptive images that efficiently encode global properties of the surface.

\subsection{Curvature Spin Images}

CSIs are created based on the ratio of surface curvatures. Let us briefly explain how to create the CSIs.

First, the reliable curvatures are extracted at 3D points sampled from the surface model $\boldsymbol{f}_{k}(u, v)$ in each gaze domain. After that, the 3D point where the absolute value of the curvature is the maximum is searched. Let us call the searched point a reference point $\boldsymbol{P}$, and its curvature a reference curvature $\kappa_{c}$. Since we can obtain the $K$ reference points, we define new $K$ gaze

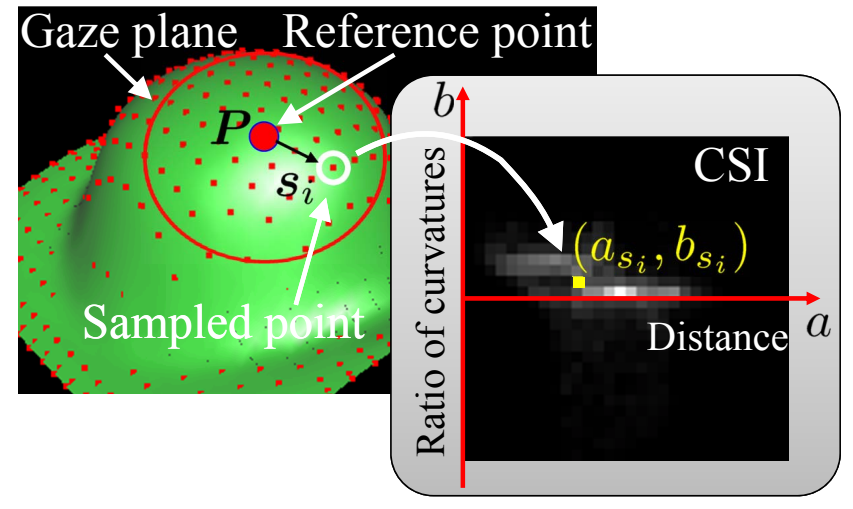

Fig. 3: The example of CSI created from a surface model.

domains whose center points are corresponding to the reference points.

Next, the CSIs are created for the $K$ reference points $\boldsymbol{P}$ by using the reference curvatures $\kappa_{c}$ as follows. The CSI is a $2 \mathrm{D}$ image that has coordinates $(a, b)$. The coordinate $a$ is defined as the distance from the reference point $\boldsymbol{P}$. The coordinate $b$ is defined as the ratio of a curvature to the reference curvature $\kappa_{c}$. A 2D accumulator indexed by $a$ and $b$ is created.

Now, the coordinates $\left(a_{s_{i}}, b_{s_{i}}\right)$ are computed for all $3 \mathrm{D}$ points $\boldsymbol{s}_{i}$ sampled from the surface model $\boldsymbol{f}_{k}(u, v)$ in the $k$ th gaze domain. $a_{s_{i}}$ can be easily computed as the distance from the reference point $\boldsymbol{P}$ to the point $\boldsymbol{s}_{i}, a_{s_{i}}=\left\|\boldsymbol{s}_{i}-\boldsymbol{P}\right\| . b_{s_{i}}$ can be simply computed as the ratio of the curvature $\kappa_{s_{i}}$ at the point $\boldsymbol{s}_{i}$ to the reference curvature $\kappa_{c}$. However, in order to acquire more large dynamic range, $b_{s_{i}}$ is computed using logarithm as follows,

$$
b_{s_{i}}=\operatorname{sgn}\left(\frac{\kappa_{s_{i}}}{\kappa_{c}}\right)|\log | \frac{\kappa_{s_{i}}}{\kappa_{c}}||+b_{o},
$$

where $b_{o}$ denotes an offset factor. It is used to adjust the range that we should focus on.

Finally, the bin indexed by $\left(a_{s_{i}}, b_{s_{i}}\right)$ in the accumulator is then incremented; bilinear interpolation is used to smooth the contribution of the sample point. This procedure is repeated for all sample points that are within the $k$ th gaze domain. The resulting accumulator can be thought of as an image; bright areas in the image correspond to bins that contain many projected points. Consequently, the CSI describes how curvature changes as the distance between the sample point and the reference point becomes large.

\section{Experimental Results}

\subsection{Experimental outline}

In this section, we will show the experimental outline. In this experiment, we consider about influence of the gaze radius, the kind of curvature, and image size by generated CSI for nine 3D models, and compare 
CSI to each model. Those Nine 3D models are head of golf club, fossil of dinosaur(whole body), bone of human hand, horse, rabbit, driver, vase, head of stone figure and rocker arm(part of engine). We choose vase as benchmark. Fig. 4 shows the example of models.

In Gaze modeling, gaze domains cover about $80 \%$ of all data points to each model. The number of gaze domain is adjusted to satisfy this. Gaze radius sets one-sixth, one-eighth, and one-tenth of size of 3D shape to each model.

In generating CSI, image size sets $20 \times 20,40 \times 20,20$ $\times 40$ and $40 \times 40$. Type of curvature set maximum and minimum curvature, product of two of them (Gaussian curvature) and average curvature.

In comparing CIS of benchmark model to another eight models, we define a correlation coefficient as follow:

$$
R(P, Q)=\frac{N \sum p_{i} q_{i}-\sum p_{i} \sum q_{i}}{\sqrt{\left\{N \sum p_{i}^{2}-\left(\sum p_{i}\right)^{2}\right\}\left\{N \sum q_{i}^{2}-\left(\sum q_{i}\right)^{2}\right\}}} .
$$

$P$ and $Q$ denote images to each models. $p$ and $q$ denote a pixel value to each images.

\subsection{The Margin}

Margin is concept for explaining the ability of our method. It is defined as:

$$
m g_{k, l}=\frac{1}{n} \sum_{i=1}^{n}\left[\max _{j} R\left(I_{k i}, I_{k^{\prime} j}\right)-\max _{l \neq k^{\prime}} \max _{j} R\left(I_{k i}, I_{l j}\right)\right]
$$

Thus, margin is the total for correlation coefficients of the benchmark model $k$ to benchmark model $k^{\prime}$ minus total for benchmark model $k$ to another model $l$. Parameter $n$ is the number of the images, $i$ is an image number of model $k$, and $j$ is an image number of model $k^{\prime}$ or $l$. If the margin is high, the distinction ability of models is high. In this experiment, this value is assumed to be a criteria.

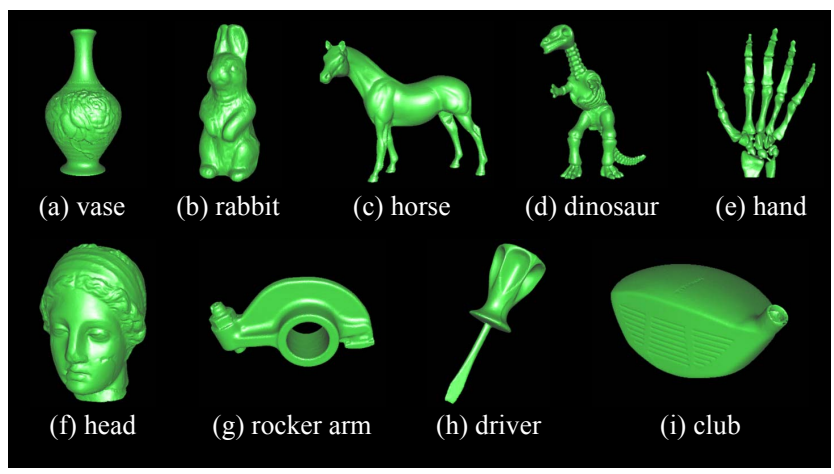

Fig. 4: Example of models.

\subsection{Result}

We experimented by the data of nine models by using the method for having proposed it so far. As an example, Figs.5 shows the example of CSIs of that experiment.

Figs.6-7 show the histogram of correlation coefficient of Gaussian and maximum curvature that is obtained by comparison of vase to vase. Figs.8-9 show vase to dinosaur. Horizontal axis shows correlation coefficient $(0.0 \sim 1.0)$, vertical axis shows number of correlation coefficient, and color of bar shows image size.

For Figs.5, some difference of each shape of the objects was seems in CSI, it seemed that there was a possibility of containing an effective feature.

For Figs.6-9, there is a difference in the distribution of correlation coefficient by difference of parameters or models. Distribution of Gaussian's correlation coefficient was lower, maximum s correlation coefficient was larger than other three curvature. Distribution of minimum and average lay midway between Gaussian and maximum.
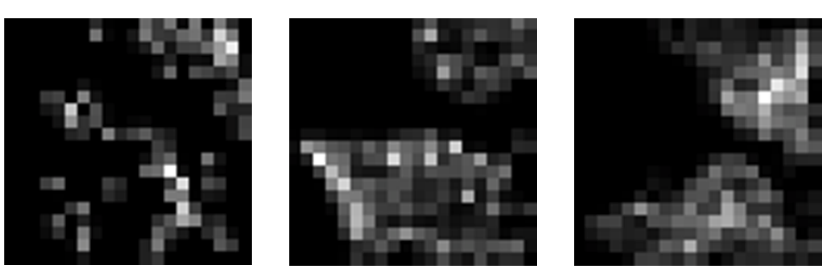

Fig. 5: Some example of CSI's results.

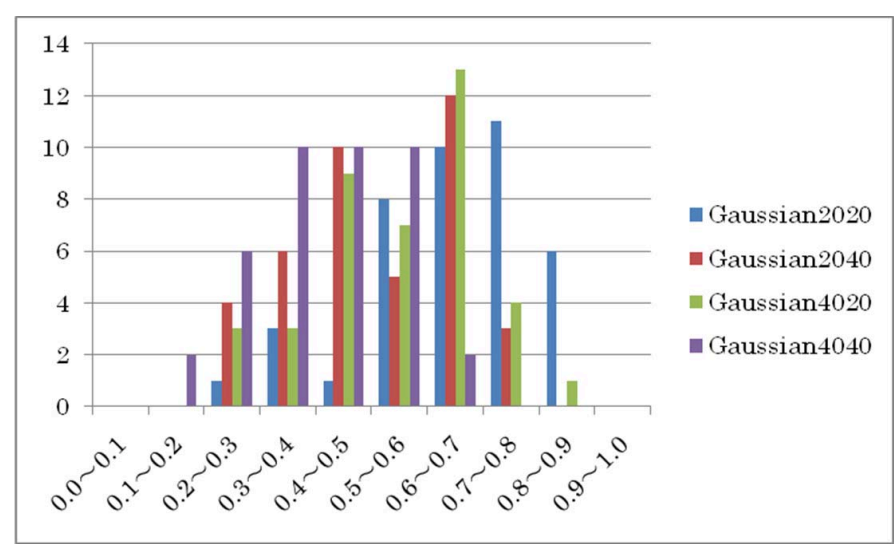

Fig. 6: Histogram of correlation coefficient of Gaussian curvature (vase to vase).

Next, we calculated margin of resultant correlation coefficient of benchmark model to another eight models. As an example, a number of margins is shown Tables 14. Those margin show that compare vase to dinosaur, and vase to hand. In Tables 1-2, margin were calculated based on CSI that did not revise. In Tables 3-4, margin were calculated based on CSI that was revised distribution of pixel value that came to the center of the image(offset). 


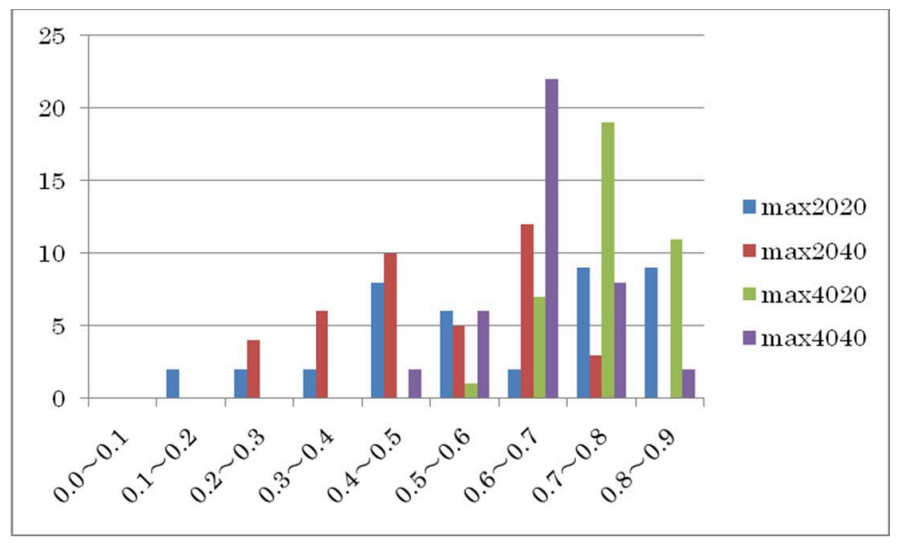

Fig. 7: Histogram of correlation coefficient of maximum curvature (vase to vase).

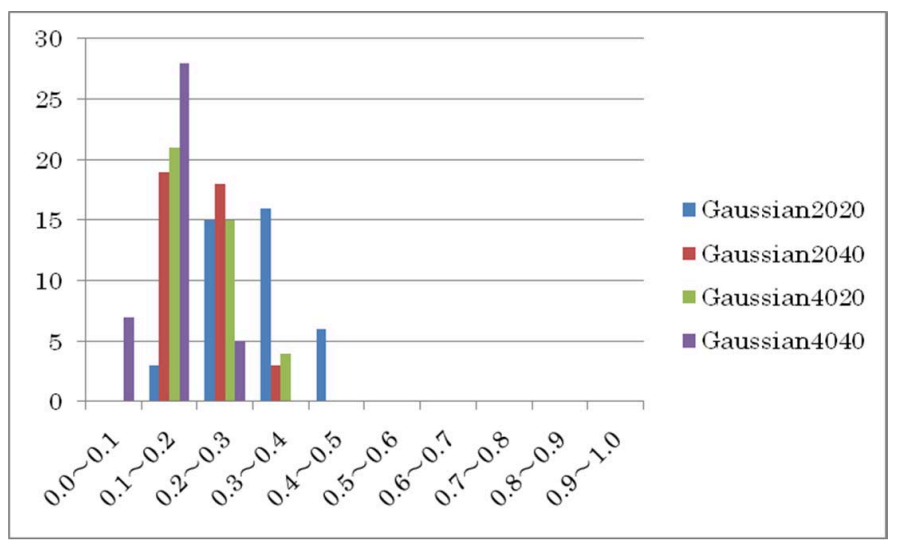

Fig. 8: Histogram of correlation coefficient of Gaussian curvature (vase to dinosaur).

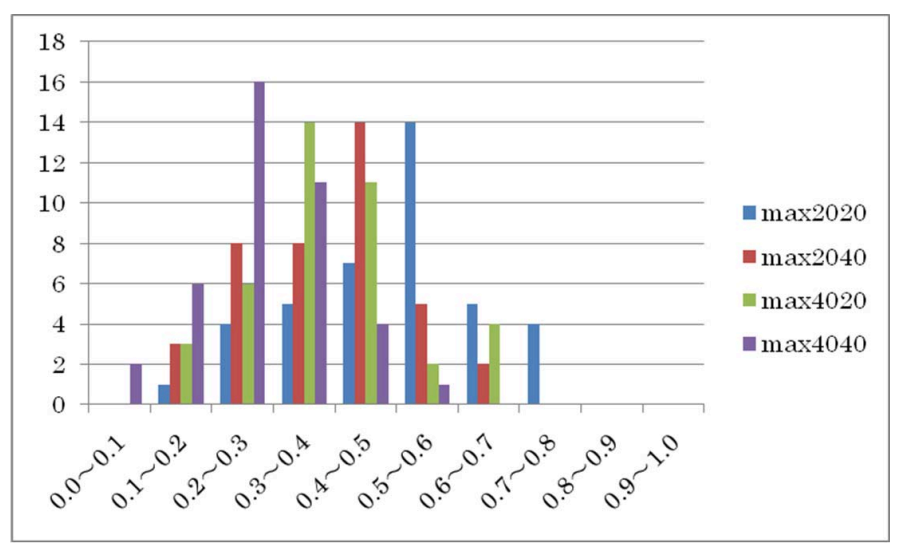

Fig. 9: Histogram of correlation coefficient of maximum curvature (vase to dinosaur).

First, we focus the curvature. We calculated margin of resultant correlation coefficient of benchmark model to another eight models. As an example, a number of margins is shown Tables 1-4. Those margin show that compare vase to dinosaur, and vase to hand. In Tables 1-2, margin were calculated based on CSI that did not revise. In Tables 3-4, margin were calculated based on CSI that was revised distribution of pixel value that came to the center of the image(offset).

For Tables 1-2, when compared margin to each curvatures, the largest value was Gaussian's margin. In contrast, maximum's was lowest of four curvatures. But, In Tables 3-4, Gaussian's margin was decreased, maximum's and average's margin were increased. Minimum's margin did not really change. In the comparison with the other models, a similar tendency was seen.

For this result, expected increase of margin were obtained from maximum and average by offsetting. But, Gaussian and minimum margin were not. This reason may be related to distribution of the pixel in CSI. may be used as an effective parameter. In any case, if CSI was not offset, Gaussian curvature have high margin. Gaussian and maximum curvature are assumed candidates of parameter in this experiment.

Second, we focus the image size. In general, the image size is larger, the correlation coefficient is smaller. But, the clear difference was not obtained. Effective image size is set $20 \times 20$ temporarily.

Table 1: Margin of correlation coefficient (vase to dinosaur).

\begin{tabular}{|c|c|c|c|c|}
\hline & $20 \times 20$ & $40 \times 20$ & $20 \times 40$ & $40 \times 40$ \\
\hline Gaussian & 0.360 & 0.348 & 0.310 & 0.273 \\
\hline Mean & 0.147 & 0.179 & 0.129 & 0.150 \\
\hline Max & 0.100 & 0.128 & 0.076 & 0.098 \\
\hline Min & 0.180 & 0.216 & 0.172 & 0.184 \\
\hline
\end{tabular}

Table 2: Margin of correlation coefficient (vase to hand).

\begin{tabular}{|c|c|c|c|c|}
\hline & $20 \times 20$ & $40 \times 20$ & $20 \times 40$ & $40 \times 40$ \\
\hline Gaussian & 0.265 & 0.283 & 0.243 & 0.228 \\
\hline Mean & 0.078 & 0.110 & 0.075 & 0.095 \\
\hline Max & -0.049 & -0.018 & -0.069 & -0.033 \\
\hline Min & 0.079 & 0.110 & 0.075 & 0.095 \\
\hline
\end{tabular}

Table 3: Margin of correlation coefficient (vase to dinosaur, offset).

\begin{tabular}{|c|c|c|c|c|}
\hline & $20 \times 20$ & $40 \times 20$ & $20 \times 40$ & $40 \times 40$ \\
\hline Gaussian & 0.245 & 0.253 & 0.184 & 0.176 \\
\hline Mean & 0.237 & 0.243 & 0.242 & 0.247 \\
\hline Max & 0.305 & 0.365 & 0.333 & 0.380 \\
\hline Min & 0.161 & 0.207 & 0.160 & 0.199 \\
\hline
\end{tabular}

Finally, we tested about gaze radius. Curvatures were set Gaussian and maximum, image size were set $20 \times$ 20 from past results. 
Table 4: Margin of correlation coefficient (vase to hand, offset).

\begin{tabular}{|c|c|c|c|c|}
\hline & $20 \times 20$ & $40 \times 20$ & $20 \times 40$ & $40 \times 40$ \\
\hline Gaussian & 0.176 & 0.195 & 0.131 & 0.133 \\
\hline Mean & 0.200 & 0.204 & 0.189 & 0.202 \\
\hline Max & 0.230 & 0.293 & 0.221 & 0.285 \\
\hline Min & 0.061 & 0.117 & 0.081 & 0.138 \\
\hline
\end{tabular}

For Tables 5-6, when curvature is Gaussian, small gaze radius had high margin. When curvature is maximum, large radius had large margin. This result show that maximum curvature is effective, because large gaze radius dose not have problem when there is only one model in data space. But, it is necessary that gaze radius is small when there are any models in data space. For instance, Gaussian curvature is effective.

Table 5: Margin of gaze radius (maximum).

\begin{tabular}{|c|c|c|c|}
\hline & $1 / 6$ & $1 / 8$ & $1 / 10$ \\
\hline club & 0.311 & 0.27 & 0.191 \\
\hline driver & 0.397 & 0.39 & 0.217 \\
\hline horse & 0.202 & 0.113 & 0.085 \\
\hline
\end{tabular}

Table 6: Margin of gaze radius (Gaussian).

\begin{tabular}{|c|c|c|c|}
\hline & $1 / 6$ & $1 / 8$ & $1 / 10$ \\
\hline club & 0.185 & 0.442 & 0.425 \\
\hline driver & 0.204 & 0.46 & 0.462 \\
\hline horse & 0.201 & 0.244 & 0.129 \\
\hline
\end{tabular}

\section{Conclusion}

This paper proposed a new approach for object recognition, gaze modeling and CSI. We consider about influence of the gaze radius, the kind of curvature, and image size by generated CSI

First experimental results showed that effective curvature might be Gaussian curvature. Concerning image size, we did not obtain a clear difference. Second experiment result showed that superiority of two curvature changed by the situation of models.

From above-mentioned result, we understood the part of the tendency of each parameters. But,there results are not enough, the unidentified parameter is still left. We must conduct more experiment while reviewing parameter and comparison method.

\section{References}

[1] Andrew E. Johnson and Martial Hebert, "Using Spin Images for Efficient Object Recognition in
Cluttered 3D Scenes," IEEE Trans. Pattern Analysis and Machine Intelligence, Vol. 21, No. 5, pp. 433-449, 1999.

[2] F. Mokhtarian, N. Khalili and P. Yuen, "Multiscale free-form 3D object recognition using 3D models," Image and Vision Computing, Vol. 19, pp. 271-281, 2001.

[3] A. Goetz, "Introduction to Differential Geometry, " Addison-Wesley, Reading, MA, 1970.

[4] M. Maeda, K. Kumamaru and K. Inoue, "Shapes Modeling of 3-D Objects Based on a Hybrid Representation Using Extended B-spline Surface Model," 16th International Conference on Pattern Recognition(ICPR2002) pp.656-659, 2002. 